\title{
A synthesis of some new 4-arylidene-5(4H)-oxazolone azo dyes and an evaluation of their solvatochromic behaviour
}

\author{
Samieh Fozooni, ${ }^{\text {a }}$ Ahmad Momeni Tikdari, ${ }^{\text {a } * \text { Hooshang Hamidian, }}{ }^{\text {b }}$ \\ and Hojatollah Khabazzadeh ${ }^{\mathrm{a}}$ \\ ${ }^{a}$ Department of Chemistry, Shahid Bahonar University of Kerman, Kerman, 76135-133, Iran \\ ${ }^{b}$ Department of Chemistry, Payame Noor University (PNU), Kerman, Iran \\ E-mail: amomeni_uk@yahoo.com
}

\begin{abstract}
Nine novel 4-arylidene-5(4H)-oxazolone azo dyes were synthesized in good yield by reacting sodium 2-[4-\{2-[4-(dimethylamino)phenyl]-1-diazenyl $\}$ benzoylamino]acetate with suitable aldehydes in the presence of $\mathrm{Ph}_{3} \mathrm{P} / \mathrm{CCl}_{4}$ and acetonitrile at room temperature. Sodium 2-[4-\{2-[4(dimethylamino)phenyl]-1-diazenyl benzoylamino]acetate was obtained by diazotization of 4aminohippuric acid and coupling with $N, N$-dimethylaniline. These dyes were characterized by elemental and spectral analyses and the solvatochromic behaviour in various solvents was evaluated.
\end{abstract}

Keywords: $5(4 H)$-Oxazolone azo dyes, solvents' effect, diazotization, synthesis, $\mathrm{Ph}_{3} \mathrm{P} / \mathrm{CCl}_{4}$, acetonitrile

\section{Introduction}

Many heterocyclic compounds are used extensively in disperse dye chemistry for textile or nontextile applications. These dyes are now marketed to produce a full range of dispersed dyestuffs without the use of colorants based on heteroaromatic diazo components. Most of the heterocyclic dyes are derived from the diazo components consisting of five-membered rings containing one or more nitrogen heteroatoms, with the rings being fused into another aromatic ring. 1,2

The azo dyes containing heterocyclic rings result in brighter and often deeper shades than their benzene analogs. On the other hand, they are very important in applications such as disperse dyes for polyester fibers, reprography, functional dye and nonlinear optical systems, photodynamic therapy, and lasers. $^{3-9}$

The 5(4H)-oxazolones are important synthons for the synthesis of several biologically active molecules. Also, they are precursors of amino acids containing an aromatic side chain. A number 
of methods are available for the synthesis of azlactones including the use of acetic anhydride and sodium acetate, acetic anhydride and lead acetate, polyphosphoric acid, sulfur trioxide/dimethyl formamide complex, perchloric acid, and carbodiimides. Recently, synthesis of oxazolones has been reported by using anhydrous zinc chloride or bismuth (III) acetate as catalysts. ${ }^{10-15}$.

It is well known that triphenylphosphane in combination with tetrachloromethane provides reagents that have manifold uses and are finding increasing application in preparative chemistry for halogenation, dehydration, and $\mathrm{P}-\mathrm{N}$ linking reagents. Of more general importance is the tertiary phosphane/tetrachloromethane system, as chlorinating and dehydrating agent for sensitive substrates to the aggressive and readily hydrolyzed acid chlorides such as $\mathrm{PCl}_{5}$, $\mathrm{P}(\mathrm{O}) \mathrm{Cl}_{3}$, thionyl chloride and sulfonyl chloride. A great advantage can also be seen in its ability to the demands made by the various donor strengths of the substituents chosen for attachment to the phosphorus atom. ${ }^{16-18}$

Herein, we wish to report a novel method for synthesis of some new 4-arylidene-5(4H)oxazolone azo dye derivatives from sodium 2-[4-\{2-[4-(dimethylamino)phenyl]-1-diazenyl $\}$ benzoylamino]acetate and corresponding aldehydes in the presence of $\mathrm{PPh}_{3}$ and $\mathrm{CCl}_{4}$ at room temperature.

\section{Results and Discussion}

Sodium 2-[4-\{2-[4-(dimethylamino)phenyl]-1-diazenyl $\}$ benzoyl amino] acetate was prepared by diazotization of 4-aminohippuric acid in nitrosyl hydrochloric acid followed by coupling with $N, N$-dimethylaniline to give yellow crystals in good yield. Then 4-arylidene-5(4H)-oxazolone azo dyes (3a-i) were synthesized by classical Erlenmeyer reaction, involving condensation of sodium 2-[4-\{2-[4-(dimethylamino)phenyl]-1-diazenyl $\}$ benzoylamino] acetate with corresponding aldehydes in presence of acetic anhydride and sodium acetate under refluxing condition (method $\mathrm{A}$ ). In method $\mathrm{B}$, We found that $\mathrm{Ph}_{3} \mathrm{P} / \mathrm{CCl}_{4}$ reagent easily converted the compound 2 to 4-arylidene-5(4H)-oxazolone azo dyes (3a-i) under refluxing with corresponding aldehydes and $\mathrm{NEt}_{3}$ in $\mathrm{CCl}_{4}$. In this investigation, at first triphenylphosphane reacts with carbon tetrachloride to give triphenylphosphonium chloride. Next compound $\mathbf{A}$ is formed along with the generation of triphenylphosphane oxide. Then aromatic aldehydes are condensed with $\mathbf{A}$ to give the 4-arylidene-5(4H)-oxazolone azo dye products. A reasonable mechanism is shown in Scheme 1. In method $\mathrm{C}$, we investigated the effect of acetonitrile as a solvent on the $\mathrm{Ph}_{3} \mathrm{P} / \mathrm{CCl}_{4}$ reaction. It was seen that with $\mathrm{Ph}_{3} \mathrm{P} / \mathrm{CCl}_{4} / \mathrm{MeCN}$ an excellent yield of highly pure product was obtained at room temperature and short time whereas two previous methods were carried out at high temperature, long time and moderate yields (Table1). The choice of acetonitrile was based on a

review by Appel $^{19,20}$ where it was pointed out that acetonitrile can have marked accelerating effect on $\mathrm{Ph}_{3} \mathrm{P} / \mathrm{CCl}_{4}$ reactions, owing to its high dielectric constant and solvating ability compared to $\mathrm{CCl}_{4}$ itself. Other merit of this method is for sensitive substrates to hydrolyze in the acidic solution. 

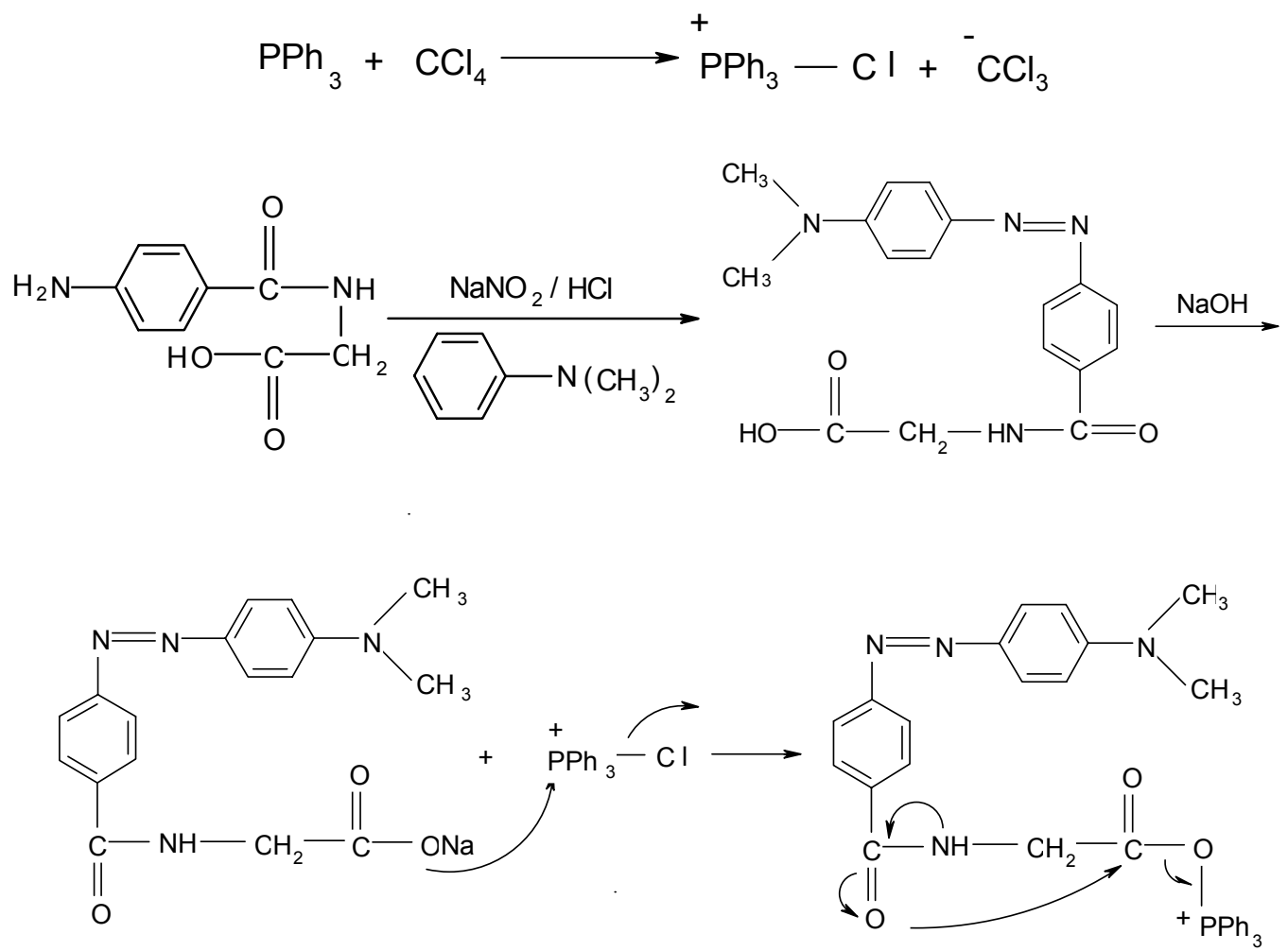

2

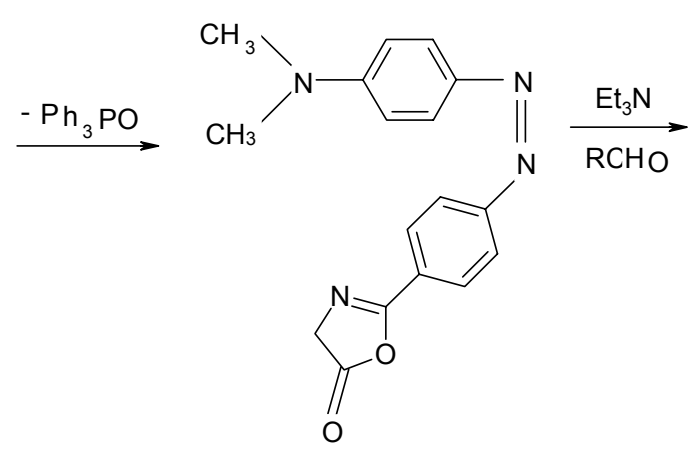

A

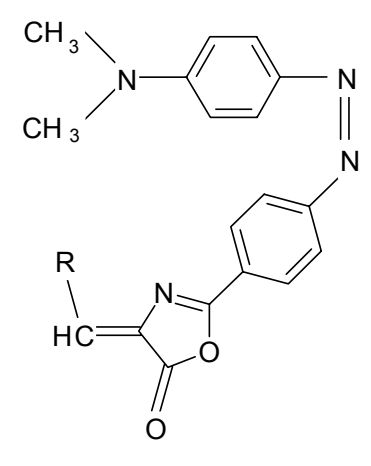

3

3a: $\mathrm{R}_{1}=\mathrm{C}_{6} \mathrm{H}_{5}$

3b: $\mathrm{R}_{2}=4-\mathrm{ClC}_{6} \mathrm{H}_{4}$

3c: $\mathrm{R}_{3}=2,4-\mathrm{Cl}_{2} \mathrm{C}_{6} \mathrm{H}_{3}$

3d: $\mathrm{R}_{4}=4-\mathrm{MeOC}_{6} \mathrm{H}_{4}$

3e: $\mathrm{R}_{5}=2-\mathrm{MeOC}_{6} \mathrm{H}_{4}$

3f: $\mathrm{R}_{6}=4-\mathrm{MeC}_{6} \mathrm{H}_{4}$

3g: $\mathrm{R}_{7}=3-\mathrm{O}_{2} \mathrm{NC}_{6} \mathrm{H}_{4}$

3h: $\mathrm{R}_{8}=\mathrm{C}_{4} \mathrm{H}_{3} \mathrm{O}$

3i: $\mathrm{R}_{9}=\mathrm{C}_{4} \mathrm{H}_{3} \mathrm{~S}$

\section{Scheme 1}


Table1. Yield (\%) of dyes 3a-i by three methods

\begin{tabular}{cccc}
\hline Products & Method A & Method B & Method C \\
\hline 3a & 78 & 80 & 85 \\
3b & 67 & 72 & 78 \\
3c & 78 & 83 & 90 \\
3d & 66 & 70 & 75 \\
3e & 59 & 63 & 70 \\
3f & 65 & 69 & 74 \\
3g & 76 & 80 & 86 \\
3h & 85 & 89 & 92 \\
3i & 75 & 78 & 83 \\
\hline
\end{tabular}

The absorption spectra of these oxazolone azo dyes 3a-i were recorded in various solvents at the concentration of $10^{-6} \mathrm{M}$, and the results are given in Table 2 . We found that the electronic absorption of these oxazolone azo dyes indicated a regular variation with the polarity of solvents, which did not change significantly. These dyes, apparently, did not exhibit a strong solvent dependence. The maximum absorption of these dyes shifted in the order: DMF $>$ acetone $>$ chloroform. The spectral shifts of dye 3a in various solvents are shown in Fig 1. The maximum absorption of dye 3a showed bathochromic shift in DMF and acetone, with respect to the maximum absorption in chloroform (e.g. $\lambda_{\max }$ is $494 \mathrm{~nm}$ in DMF, $492 \mathrm{~nm}$ in acetone, and $490 \mathrm{~nm}$ in chloroform). The same trends of absorption shifts in various solvents were observed for the entire series of dyes 3a-i, as shown in Table 2. The substituent effects of the heterocyclic azo dyes 3a-i were evaluated. The spectral shifts of dyes $\mathbf{3 a}-\mathbf{i}$ in chloroform at a concentration of $10^{-}$ ${ }^{6}$ are given in Table 3 . We found that the dyes $\mathbf{3 d}$ and $\mathbf{3 e}$ contain an electrondonor group methoxy on the 4th and 2nd position of phenyl group substituted on the 4th position of oxazolone ring, so that the $\lambda_{\max }$ of dye $\mathbf{3 d}$ and $\mathbf{3 e}$ showed hypsochromic shift of -6 and -2 relative to the dye 3a, respectively.

Dye 3g contains an electron acceptor group (nitro) in the 3rd position of the phenyl group substituted on the 4 th position of the oxazolone ring, so that the $\lambda_{\max }$ of dye $3 \mathrm{~g}$ showed bathochromic shift of $+13 \mathrm{~nm}$ relative to $\mathbf{3} \mathbf{a}, \lambda_{\max }$ of dyes $\mathbf{3 b}$ and $\mathbf{3} \mathbf{c}$ is $+4 \mathrm{~nm}$ and $+10 \mathrm{~nm}$ longer than that of dye 3a, due to weaker electron acceptor of chloro group in the para and ortho, para positions of phenyl group substituted on the 4 th position of oxazolone ring, respectively, $\lambda_{\max }$ of dye $3 \mathbf{h}$ showed bathochromic shift of $+3 \mathrm{~nm}$ relative to $\mathbf{3 a}$ and $\lambda_{\max }$ of dye $\mathbf{3 i}$ is $+4 \mathrm{~nm}$ longer than that of dye $\mathbf{3 a}$. 


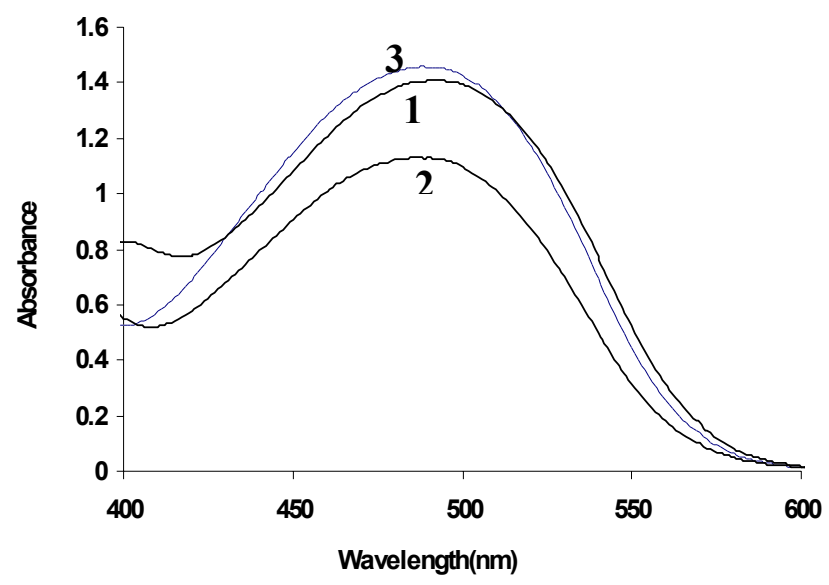

Figure 1. Absorption spectra of dye 1. DMF; 2. acetone; 3. chloroform.

Table 2. Absorption spectra of dyes 3a-i in various solvents

\begin{tabular}{cccc}
\hline Products & DMF & Acetone & Chloroform \\
\hline 3a & 494 & 492 & 490 \\
3b & 499 & 498 & 494 \\
3c & 506 & 503 & 500 \\
3d & 490 & 488 & 484 \\
3e & 493 & 490 & 488 \\
3f & 496 & 494 & 492 \\
3g & 511 & 506 & 503 \\
3h & 506 & 499 & 493 \\
3i & 506 & 500 & 494 \\
\hline
\end{tabular}

The $\varepsilon_{\mathrm{r}}$ value of solvents: $\mathrm{DMF}=36.7$; chloroform $=4.8$.

Table 3. Substituent effect of dyes 3a-i in chloroform

\begin{tabular}{ccccc}
\hline Products & $\lambda_{\max }$ & $\mathrm{R}$ & $\Delta \lambda^{\mathrm{a}}$ & $\log \varepsilon$ \\
\hline 3a & 490 & $\mathrm{C}_{6} \mathrm{H}_{5}$ & - & 6.16 \\
3b & 494 & $4-\mathrm{ClC}_{6} \mathrm{H}_{4}$ & +4 & 6.11 \\
3c & 500 & $2,4-\mathrm{Cl}_{2} \mathrm{C}_{6} \mathrm{H}_{3}$ & +10 & 6.01 \\
3d & 484 & $4-\mathrm{MeOC}_{6} \mathrm{H}_{4}$ & -6 & 6.05 \\
3e & 488 & $2-\mathrm{MeOC}_{6} \mathrm{H}_{4}$ & -2 & 6.17 \\
3f & 492 & $4-\mathrm{MeC}_{6} \mathrm{H}_{4}$ & +2 & 6.05 \\
3g & 503 & $3-\mathrm{O}_{2} \mathrm{NC}_{6} \mathrm{H}_{4}$ & +13 & 6.01 \\
3h & 493 & $\mathrm{C}_{4} \mathrm{H}_{3} \mathrm{O}$ & +3 & 6.08 \\
3i & 494 & $\mathrm{C}_{4} \mathrm{H}_{3} \mathrm{~S}$ & +4 & 6.14 \\
\hline
\end{tabular}

${ }^{\mathrm{a}}$ Relative to 3a. 


\section{Experimental Section}

General Procedures. Melting points were determined on a Gallenkamp melting point apparatus and are uncorrected. IR spectra were recorded with the MATTSON 1000 FT-IR spectrophotometer. Nuclear magnetic resonance spectra were recorded on the BRUKER DRX500 AVANCE spectrometer using tetramethylsilane (TMS) as an internal standard. Mass spectra were obtained by SHIMADZU QP 1100EX. Elemental analyses were performed by the Iranian Oil Company using a Heracus CHN-O-Rapid analyzer. UV spectra were recorded using a GBC Scientific Equipment (CINTRA 5) UV visible spectrometer.

\section{General preparative procedures}

Sodium 2-[4-\{2-[4-(dimethylamino)phenyl]-1-diazenyl $\}$ benzoyl amino] acetate. In a 125$\mathrm{mL}$ Erlenmeyer flask, 4-aminohippuric acid $(0.01 \mathrm{~mol})$ was added to $2 \%$ sodium carbonate solution $(30 \mathrm{~mL})$ until it was dissolved by boiling. The solution was then cooled and sodium nitrite $(0.01 \mathrm{~mol})$ was added, with stirring, until it was dissolved. The solution was cooled by placing it in an ice bath, and then concentrated hydrochloric acid $(2 \mathrm{~mL})$ and water $(3 \mathrm{~mL})$ were added. By acidifying the solution, a powdery yellow precipitate of the diazonium salt was separated.

$N, N$-Dimethylaniline $(0.01 \mathrm{~mol})$ and glacial acetic acid $(0.01 \mathrm{~mol})$ was mixed. The solution of $N, N$-dimethylaniline acetate was added to suspension of diazotized hippuric acid, with stirring, and acid-stable form of the dye was separated. A stiff paste was formed in 5-10 min and then sodium hydroxide $(5 \mathrm{~g})$ was added to produce the orange sodium salt. The product was collected using saturated sodium chloride solution. The crude product was crystallized from water (2). Orange crystals, decomposed $>270$ yield is $81 \%$. IR $(\mathrm{KBr}): \mathrm{v}=3354,1716 \mathrm{~cm}^{-1} .{ }^{1} \mathrm{H}$ NMR $(500$ MHz, DMSO-d $)_{6}$ : 3.07 (s, 6H, 2CH 3 ), 3.61 (d, 2H, J 4.4 Hz, $\mathrm{CH}_{2}$ ), 6.84 (d, 2H, J 8.9 Hz, ArH), 7.80-7.82 (m, 4H, ArH), 7.92 (broad, 1H, NH), 7.98 (d, 2H, J 8.5, ArH) ppm. ${ }^{13} \mathrm{CNMR}(500$ MHz, DMSO-d 6 ): 40.69, 112.44, 122.46, 125.93, 128.94, 136.03, 143.52, 153.67, 154.69. $\mathrm{C}_{17} \mathrm{H}_{17} \mathrm{O}_{3} \mathrm{~N}_{4} \mathrm{Na}$ (348.33) calcd. C 58.65, H 4.88, N 16.08; found. C 58.54, H 4.92, N 16.18.

Method A. Preparation of 4-arylidene-5(4H)-oxazolone azo dyes with Erlenmeyer method A mixture of finely divided, anhydrous sodium acetate $(0.01 \mathrm{~mol})$, appropriate aldehyde $(0.01 \mathrm{~mol}), \quad$ sodium 2-[4-\{2-[4-(dimethylamino)phenyl]-1-diazenyl $\}$ benzoylamino] acetate $(0.01 \mathrm{~mol})$, and acetic anhydride $(40 \mathrm{~mL})$ was heated with intermittent shaking until the mixture is transformed from an orange, semi-solid mass to a deep red liquid $(2 \mathrm{~h})$. The mixture was then cooled to room temperature and the crystalline product was separated by filtration. The crude product was recrystallized from toluene.

Method B. Preparation of 4-arylidene-5(4H)-oxazolone azo dyes with $\mathbf{P P h}_{\mathbf{3}} / \mathrm{CCl}_{4}$. A mixture of sodium 2-[4-\{2-[4-(dimethylamino)phenyl]-1-diazenyl $\}$ benzoylamino] acetate $(0.01 \mathrm{~mol})$, $\mathrm{PPh}_{3}(0.01 \mathrm{~mol})$, corresponding aldehyde $(0.01 \mathrm{~mol})$ and $\mathrm{NEt}_{3}(1 \mathrm{~mL})$ was dissolved in $\mathrm{CCl}_{4}(25$ $\mathrm{mL}$ ). The solvent was evaporated after the reaction system was refluxed for $6 \mathrm{~h}$. The residue was 
precipitated in water and ethanol and then was purified with toluene to give the corresponding 4arylidene-5(4H)-oxazolone azo dye products.

Method C. Preparation of 4-arylidene-5(4H)-oxazolone azo dyes with $\mathrm{PPh}_{\mathbf{3}} / \mathrm{CCl}_{\mathbf{4}}$ in acetonitrile. A mixture of sodium 2-[4-\{2-[4-(dimethylamino)phenyl]-1-diazenyl $\}$ benzoyl amino] acetate $(0.01 \mathrm{~mol}), \mathrm{PPh}_{3}(0.01 \mathrm{~mol}), \mathrm{CCl}_{4}(0.01 \mathrm{~mol}, 1 \mathrm{~mL})$, the corresponding aldehyde $(0.01 \mathrm{~mol})$ and $\mathrm{NEt}_{3}(1 \mathrm{~mL})$ was dissolved in acetonitrile $(25 \mathrm{~mL})$. The solvent was evaporated after the reaction system was stirred at room temperature for 30 minutes. The residue was precipitated in water and ethanol and then was purified with toluene to give the corresponding 4arylidene-5(4H)-oxazolone azo dye products.

2-(4-\{2-[4-(Dimethylamino)phenyl]-1-diazenyl\}phenyl)-4-[1-phenylmethylidene]-5(4H) oxazolone (3a). Dark red crystals. m.p.: $234-235^{\circ} \mathrm{C}$. yield $(85 \%)$. IR $(\mathrm{KBr}): \mathrm{v}=1815,1716 \mathrm{~cm}^{-1}$ . ${ }^{1} \mathrm{H}$ NMR (500 MHz, DMSO-d $)$ ): 3.17 (s, 6H, 2CH3), 6.81 (d, 2H, J 8.9 Hz, ArH), 7.30 (s, 1H, J $8.9 \mathrm{~Hz}$, Vinyl), 7.50-7.56 (m, 3H, ArH), 7.97 (d, 2H, J $8.8 \mathrm{~Hz}, \mathrm{ArH}), 8.02$ (d, 2H, J 8.4 Hz, ArH), $8.28(\mathrm{~d}, 2 \mathrm{H}, J 7.3 \mathrm{~Hz}, \mathrm{ArH}), 8.33$ (d, 2H, J 8.4 Hz, ArH) ppm. ${ }^{13} \mathrm{CNMR}(500$ MHz,DMSO-d $\left.\mathrm{d}_{6}\right)$ : 40.76, 111.77, 122.46, 125.93, 126.02, 129.25, 29.57, 131.55, 131.83, 132.75, 133.66, 133.82, 153.40, 156.46, 163.44. $\mathrm{C}_{24} \mathrm{H}_{20} \mathrm{~N}_{4} \mathrm{O}_{2}$ (396.429) calcd: C 72.75, H 5.05, N, 14.13; found: C 72.69, H 5.01, N 14.19. MS m/z: 396 (11\%, $\left.\mathrm{M}^{+}\right), 368$ (25\%), 224 (40\%), 148 (62\%), $120(100 \%)$.

4-[1-(4-Chlorophenyl)methylidene]-2-(4-\{2-[4-(dimethylamino)phenyl]-diazenyl $\}$ phenyl)5(4H)-oxazolone (3b). Dark brown crystals. m.p.: 260-261 ${ }^{\circ} \mathrm{C}$. yield (78\%). IR (KBr): v = 1815, $1716 \mathrm{~cm}^{-1} .{ }^{1} \mathrm{H}$ NMR (500 MHz, DMSO-d 6$): 3.17\left(\mathrm{~s}, 6 \mathrm{H}, 2 \mathrm{CH}_{3}\right), 6.81(\mathrm{~d}, 2 \mathrm{H}, J 9.1 \mathrm{~Hz}, \mathrm{ArH})$, 7.23 (s, 1H, Vinyl), 7.51 (d, 2H, J 8.6 Hz, ArH), 7.97 (d, 2H, J 9.1 Hz, ArH), 8.01 (d, 2H, J 8.6 $\mathrm{Hz}, \mathrm{ArH}), 8.21$ (d, 2H, J $8.5 \mathrm{~Hz}, \mathrm{ArH}), 8.32$ (d, 2H, J 8.6 Hz, ArH) ppm. ${ }^{13} \mathrm{CNMR}(500 \mathrm{MHz}$, DMSO-d $)_{6}$ : 40.65, 111.96, 123.139, 125.66, 126.16, 129.66, 129.82, 130.11, 130.14, 130.23, $132.59,133.91,134.32,137.66,144.38,153.57,156.88,164.09,167.75 . \mathrm{C}_{24} \mathrm{H}_{19} \mathrm{~N}_{4} \mathrm{O}_{2} \mathrm{Cl}$ (430.875) calcd. C 66.93, H 4.41, N 13.00; found. C 66.85, H 4.52, N 13.09. MS m/z: 432 (4\%, $\mathrm{M}+2), 430\left(12 \%, \mathrm{M}^{+}\right), 402(20 \%), 224(37 \%), 148$ (65\%), 120 (100\%).

4-[1-(2,4-Dichlorophenyl)methylidene]-2-(4-\{2-[4-(dimethylamino)phenyl]-diazenyl\}phenyl) -5(4H)-oxazolone (3c). Dark brown crystals. m.p.: 247-248 ${ }^{\circ} \mathrm{C}$. yield (90\%). IR (KBr): v = 1815, $1716 \mathrm{~cm}^{-1}$. ${ }^{1} \mathrm{H}$ NMR (500 MHz, DMSO-d $)$ ): 3.17 (s, 6H, 2CH$), 6.82$ (d, 2H, J $\left.9.0 \mathrm{~Hz}, \mathrm{ArH}\right)$, 7.40 (s, 1H, Vinyl), 7.46 (d, 1H, J $8.6 \mathrm{~Hz}, \operatorname{ArH}), 7.54$ (d, 1H, J 1.8 Hz, ArH), 7.97 (d, 2H, J 9.0 $\mathrm{Hz}, \operatorname{ArH}), 8.01$ (d, 2H, J 8.5 Hz, ArH), 8.31 (d, 2H, J 8.5 Hz, ArH), 8.98 (d, 1H, J 8.5 Hz, ArH) ppm. ${ }^{13} \mathrm{CNMR}$ (500 MHz, DMSO-d 6 ): 40.65, 111.96, 123.17, 125.02, 126.22, 128.13, 128.72, 129.99, 130.22, 134.39, 135.49. $\mathrm{C}_{24} \mathrm{H}_{18} \mathrm{~N}_{4} \mathrm{O}_{2} \mathrm{Cl}_{2}$ (465.32) calcd. C 61.97, H 3.87, N 12.04; found. C 61.82, H 3.85, N 12.11. MS m/z: 468 (1\%, M+4), 466( 6\%, M+2), $464\left(10 \%, \mathrm{M}^{+}\right), 436(20 \%)$, $224(32 \%), 148(60 \%), 120(100 \%)$.

2-(4-\{2-[4-(Dimethylamino)phenyl]-1-diazenyl\}phenyl)-4-[1-(4-methoxypheny)methylidene]

- 5(4H)-oxazolone (3d). Dark red crystals. m.p.: 294-295 C. yield (75\%). IR (KBr): v $=1815$, $1716 \mathrm{~cm}^{-1} ;{ }^{1} \mathrm{H}$ NMR $\left(500 \mathrm{MHz}, \mathrm{DMSO}-\mathrm{d}_{6}\right): \delta=3.16\left(\mathrm{~s}, 6 \mathrm{H}, 2 \mathrm{CH}_{3}\right), 3.94\left(\mathrm{~s}, 3 \mathrm{H}, \mathrm{OCH}_{3}\right), 6.81(\mathrm{~d}$, 2H, J 9.3 Hz, ArH), 7.06 (d, 2H, J 8.9 Hz, ArH), 7.27 (s, 1H, Vinyl), 7.96 (d, 2H, J 10.3 Hz, 
ArH),7.99 (d, 2H, J 10.6 Hz, ArH), 8.25 (d, 2H, J 8.8 Hz, ArH), 8.30 (d, 2H, J 8.6 Hz, ArH) ppm. ${ }^{13} \mathrm{CNMR}$ (500 MHz, DMSO-d 6 ): 40.64, 55.86, 111.96, 114.99, 123.06, 126.06, 126.21, $127.18,129.47,131.84,132.08,134.99,153.47,156.49,162.67,162.69,168.33 . \mathrm{C}_{25} \mathrm{H}_{22} \mathrm{~N}_{4} \mathrm{O}_{3}$ (426.45) calcd. C 70.44, H 5.16, N 13.14; found: C, 70.35; H, 5.22; N, 13.21. MS m/z: 426 (15\%, M ), 398 (24\%), 224 (30\%), 148 (50\%), 120 (100\%).

2-(4-\{2-[4-(Dimethylamino)phenyl]-1-diazenyl\}phenyl)-4-[1-(2-methoxyphenyl) methylidene]-5(4H)-oxazolone (3e). Dark red crystals. m.p.: 264-265 C. yield (70\%). IR $(\mathrm{KBr}): v=1815,1716 \mathrm{~cm}^{-1} .{ }^{1} \mathrm{H}$ NMR (500 MHz, DMSO-d $)$ ): 3.17 (s, 6H, 2CH ), 3.97 (s, 3H, $\left.\mathrm{OCH}_{3}\right), 6.81(\mathrm{~d}, 2 \mathrm{H}, J 9.1 \mathrm{~Hz}, \mathrm{ArH}), 6.98$ (d, 1H, J $\left.8.3 \mathrm{~Hz}, \mathrm{ArH}\right), 7.16$ (t, 1H, J 7.6 Hz, ArH), 7.48 (t, 1H, J $7.8 \mathrm{~Hz}$, ArH), 7.92 (s, 1H, Vinyl), 7.97 (d, 2H, J 9.1 Hz, ArH), 8.00 (d, 2H, J 8.6 $\mathrm{Hz}, \mathrm{ArH}), 8.31$ (d, 2H, J 8.6 Hz, ArH), 8.94 (d, 1H, J 7.9 Hz, ArH) ppm. ${ }^{13} \mathrm{CNMR}(500 \mathrm{MHz}$, DMSO-d $)_{6}$ : 40.72, 56.11, 111.19, 111.93, 121.49, 123.08, 123.19, 126.11, 126.24, 129.62, $133.09,133.39,144.27,153.44,156.51,159.74,163.17,168.34,178.03 . \mathrm{C}_{25} \mathrm{H}_{22} \mathrm{~N}_{4} \mathrm{O}_{3}(426.45)$ calcd. C 70.44, H 5.16, N 13.14; found. C 70.53, H 5.06, N 13.25. MS m/z: 426 (15\%, M ), 398 (23\%), 224 (31\%), 148 (55\%), $120(100 \%)$.

2-(4-\{2-[4-(Dimethylamino)phenyl]-1-diazenyl\}phenyl)-4-[1-(4-methylphenyl)methylidene]5(4H)-oxazolone (3f). dark red crystals. m.p.: $243-244^{\circ} \mathrm{C}$. yield (74\%). IR (KBr): v $=1815$, $1716 \mathrm{~cm}^{-1} .{ }^{1} \mathrm{H}$ NMR (500 MHz, DMSO-d 6 ): 2.48 (s, 3H, $\left.\mathrm{CH}_{3}\right), 3.17$ (s, 6H, 2CH$), 6.81$ (d, 2H, $9.1 \mathrm{~Hz}, \mathrm{ArH}), 7.30$ (s, 1H, Vinyl), 7.35 (d, 2H, J 8.0 Hz, ArH), 7.97 (d, 2H, J 9.1 Hz, ArH), 8.00 (d, $2 \mathrm{H}, J 8.6 \mathrm{~Hz}, \mathrm{ArH}), 8.17$ (d, 2H, J 8.1 Hz, ArH), 8.32 (d, 2H, J 8.5 Hz, ArH) ppm. ${ }^{13} \mathrm{CNMR}$ (500 MHz, DMSO-d 6 ): 22.27, 40.72, 111.92, 123.09, 125.96, 126.13, 128.77, 129.65, 130.19, $131.44,132.32$, 132.98, 133.07, 142.53, 144.24, 153.44, 156.54, 163.16, 168.29. $\mathrm{C}_{25} \mathrm{H}_{22} \mathrm{~N}_{4} \mathrm{O}_{2}$ (410.45) calcd: C 73.19, H 5.36, N 13.65; found: C 73.09, H 5.42, N 13.54 MS m/z: 410 (8\%, $\left.\mathrm{M}^{+}\right), 382(20 \%), 224(42 \%), 148(70 \%), 120(100 \%)$.

2-(4-\{2-[4-(Dimethylamino)phenyl]-1-diazenyl\}phenyl)-4-[1-(3-nitrophenyl)methylidene]5(4H)-oxazolone $\mathbf{( 3 g )}$. red-brown crystals. m.p.: $268-269^{\circ} \mathrm{C}$. yield (86\%). IR (KBr): v $=1815$, $1716 \mathrm{~cm}^{-1} .{ }^{1} \mathrm{H}$ NMR (500 MHz, DMSO-d 6 ): $3.17\left(\mathrm{~s}, 6 \mathrm{H}, 2 \mathrm{CH}_{3}\right), 6.81(\mathrm{~d}, 2 \mathrm{H}, J 8.9 \mathrm{~Hz}, \mathrm{ArH})$, 7.24 (s, 1H, Vinyl), 7.38 (d, 1H, J 7.7 Hz, ArH), 7.51 (d, 2H, J 8.2 Hz, ArH), 7.82 (d, 1H, J 7.9 $\mathrm{Hz}, \mathrm{ArH}), 7.96-8.02$ (m, 2H, ArH), 8.22 (d, 2H, J 8.2 Hz, ArH), 8.32 (d, 2H, J 8.3 Hz, ArH) ppm. ${ }^{13} \mathrm{CNMR}$ (500 MHz, DMSO-d 6 ): 40.72, 111.94, 123.15, 125.61, 126.21, 129.69, 129.85, $130.14,130.24,132.55,133.95,134.27,137.67,144.27,153.53,156.82,167.85 . \mathrm{C}_{24} \mathrm{H}_{19} \mathrm{~N}_{5} \mathrm{O}_{4}$ (441.42) calcd: C 65.33, H 4.31, N 15.86; found: C 65.42, H 4.29, N 15.79. MS m/z: 441 (10\%, $\left.\mathrm{M}^{+}\right), 413(25 \%), 224(33 \%), 148(63 \%), 120$ (100\%).

2-(4-\{2-[4-(Dimethylamino)phenyl]-1-diazenyl\}phenyl)-4-[1-(2-furyl)methylidene]-5(4H)oxazolone (3h). red-brown crystals. m.p.: $242-243^{\circ} \mathrm{C}$. yield $(92 \%)$. IR (KBr): $v=1815,1716$ $\mathrm{cm}^{-1} .{ }^{1} \mathrm{H}$ NMR (500 MHz, DMSO-d $)$ ) 3.16 (s, 6H, 2CH 3 ), 6.71 (d, 1H, J 1.8Hz, Furyl), 6.81 (d, 2H, J 9.0 Hz, ArH), 7.22 (s, 1H, Vinyl), 7.63 (d, 1H, J 3.4 Hz, Furyl), 7.73 (s, 1H, Furyl), 7.96 (d, 2H, J 9.0 Hz, ArH), 7.99 (d, 2H, J 8.5 Hz, ArH), 8.29 (d, 2H, J 8.5 Hz, ArH) ppm. (500 MHz,

DMSO-d $)_{6}$ : ${ }^{13} \mathrm{CNMR}: 40.71,111.92,114.27,118.52,120.60,123.10,125.79,126.13,129.66$, $131.09,144.27,147.08,151.07,153.46,163.17,167.57 . \mathrm{C}_{22} \mathrm{H}_{18} \mathrm{~N}_{4} \mathrm{O}_{3}$ (386.39) calcd: $\mathrm{C} 68.42, \mathrm{H}$ 
4.66, N 14.50; found: C 68.49, H 4.68, N 14.41. MS m/z: $386\left(10 \%, \mathrm{M}^{+}\right), 224(40 \%), 148$ $(60 \%), 120(100 \%)$.

2-(4-\{2-[4-(Dimethylamino)phenyl]-1-diazenyl\}phenyl)-4-[1-(2-thienyl)methylidene]-5(4H)oxazolone (3i). Dark red crystals. m.p.: $255-256^{\circ} \mathrm{C}$. yield (83\%), IR (KBr): $v=1815,1716 \mathrm{~cm}^{-1}$ ${ }^{1} \mathrm{H}$ NMR (500 MHz, DMSO-d 6 ): 3.17 (s, 6H, 2CH3), $6.81(\mathrm{~d}, 2 \mathrm{H}, J 9.01 \mathrm{~Hz}, \mathrm{Ar}), 7.22(\mathrm{t}, 1 \mathrm{H}, J$ $4.8 \mathrm{~Hz}$, Thienyl), 7.54 (s, 1H, Vinyl), 7.69 (d, 1H, J $3.4 \mathrm{~Hz}$, Thienyl), 7.79 (d, 1H, J 5.0 Hz, Thienyl), 7.97 (d, 2H, J 8.9 Hz, ArH), 8.00 (d, 2H, $J 8.5 \mathrm{~Hz}, \mathrm{ArH}), 8.31$ (d, 2H, $J 8.5 \mathrm{~Hz}, \mathrm{ArH})$ ppm. ${ }^{13}$ CNMR (500 MHz, DMSO-d $)$ ): 40.73, 111.93, 123.13, 125.11, 125.87, 126.14, 128.40, $129.68,131.57,135.40,135.75,138.20,144.26,153.45,156.55,162.64,167.44 . \mathrm{C}_{22} \mathrm{H}_{18} \mathrm{~N}_{4} \mathrm{O}_{2} \mathrm{~S}$ (402.45) calcd: C 65.68, H 4.47, N 13.92; found: C 65.56, H 4.58, N 13.84. MS m/z: 402 (13\%, $\left.\mathrm{M}^{+}\right), 224(32 \%), 148(55 \%), 120(100 \%)$.

\section{Acknowledgements}

The authors appreciate the cooperation of the Department of Chemistry, Shahid Bahonar University of Kerman, for supporting this investigation.

\section{References and Footnotes}

1. Weaver, M. A.; Shuttleworth, L. Dyes and Pigments 1982, 3, 81.

2. Maradia, H. R.; Patel, V. S. J. Serb. Soc. 2001, 66(2), 87.

3. Towns, A. D. Dyes and Pigments 1999, 42, 3.

4. Gupta, S.; Sivasubramanian, A.; Rodrigues, L. M.; Esteves, A. P.; Hrdina, R.; OliveiraCompos, A. M. F. Dyes and Pigments 2007, 75, 82.

5. Tsai, P. C.; Wang, I. J. Dyes and Pigments 2007, 74, 578.

6. Maradiya, H. R.; Patel, V. S. J. Braz. Soc. 2001, 12, 710.

7. Razus, A. C.; Birzan, L.; Surugiu, N. M. Dyes and Pigments 2007, 74, 26.

8. Singh, K.; Singh, S.; Taylor, J. A. Dyes and Pigments 2002, 54, 189.

9. Naik, S. J.; Halkar, U. P. ARKIVOC 2005, (viii), 141.

10. Shamsunder, R. Y. Synthesis 1975, 749.

11. Paul, S.; Nanda, P.; Gupta, R.; Loupy, A. Tetrahedron Lett. 2004, 45, 425.

12. Crawford, M.; Little, W. T. J. Chem. Soc. 1959, 729

13. Beccalli, E. M.; Clerici, F.; Gelmi, M. L. Tetrahedron 1999, 55, 781.

14. Boekelheide, V.; Schramm, M. J. Org. Chem. 1949, 14, 298.

15. Kumar, P.; Mishra, H. D.; Mukerjee, A. K. Synthesis 1980, 836.

16. Yang, Y. H.; Shi, M. Tetrahedron Lett. 2005, 46, 6285.

17. Yang, Y. H.; Shi, M. Tetrahedron 2006, 62, 2420.

18. Bovin, A. N.; Chekhlov, A. N.; Svetkov, E. N. Tetrahedron Lett. 1990, 31, 5361.

19. Appel, A. Angew. Chem. Internat. Edit. 1975, 14, 801.

20. Burton, G.; Elder, J. S.; Fell, S. C. M.; Stachulaki, A. V. Tetrahedron Lett. 1988, 29, 3003. 